\title{
Bubble dynamics involved in ultrasonic imaging
}

\author{
Running title: Bubble dynamics \\ Michiel Postema, Georg Schmitz
}

Institute for Medical Engineering, Ruhr-Universität Bochum, Bochum, Germany

\begin{tabular}{l|l}
$\begin{array}{l}\text { Affiliations } \\
\text { Dr. Michiel Postema }\end{array}$ & Prof. Dr.-Ing. Georg Schmitz \\
$\begin{array}{l}\text { Institute for Medical Engineering } \\
\text { Department of Electrical Engineering }\end{array}$ & Institute for Medical Engineering \\
and Information Technology & and Information Technology \\
Ruhr-Universität Bochum & Ruhr-Universität Bochum \\
Building IC, 6/146 & Building IC, $6 / 135$ \\
D-44780 Bochum & D-44780 Bochum \\
Germany & Germany \\
Tel: +49234 3227740 & \\
Fax: +49 234 3214872 & georg.schmitz@rub.de \\
michiel.postema@rub.de
\end{tabular}


Summary - In clinical ultrasound, blood cells cannot be differentiated from surrounding tissue, due to the low acoustic impedance difference between blood cells and their surroundings. Resonant gas bubbles introduced in the blood stream are ideal markers, if rapid dissolution can be prevented. Ultrasound contrast agents consist of microscopically small bubbles encapsulated by an elastic shell. These microbubbles oscillate upon ultrasound insonification. Microbubbles with thin lipid shells have demonstrated highly nonlinear behavior. To enhance diagnostic ultrasound imaging techniques and to explore therapeutic applications, these medical microbubbles have been modeled. Several detection techniques have been proposed to improve the detectability of the microbubbles. A new generation of contrast agents, with special targeting ligands attached to the shells, may assist in imaging nonphysical properties of target tissue. Owing to microbubble-based contrast agents, ultrasound is becoming an even more important technique in clinical diagnostics.

Keywords: microbubbles, ultrasound, ultrasound contrast agent, scattering, resonance, RPNNP equation, Herring equation, harmonic imaging, targeted imaging, detection. 


\section{Bubble dynamics involved in ultrasonic imaging}

Ultrasonic imaging is a relatively cheap, reliable diagnostic technique. Typical diagnostic ultrasonic frequencies range from $1 \mathrm{MHz}$ (heart, liver) to $100 \mathrm{MHz}$ (eye, skin). A signal generated by an ultrasonic transducer typically consists of a pulse of a few $\mu$ s with an angular center frequency $\omega$. Part of this signal propagates through target tissue, part is reflected by macroscopic tissue structures, part is absorbed by tissue, and part is scattered by structures in the tissue smaller than the acoustic wavelength. A small portion of the transmitted acoustic energy is received by the transducer, which is used to build an ultrasonic image. The received signal is the superposition of specular reflections at tissue boundaries and echoes from tissue backscattering. The quantity of signal specularly reflected from a boundary separating tissues 1 and 2 depends on the acoustic impedance change in the tissue:

$$
R_{\mathrm{I}}=\frac{\left(Z_{2}-Z_{1}\right)^{2}}{\left(Z_{2}+Z_{1}\right)^{2}},
$$

where $R_{\mathrm{I}}$ is the intensity reflection coefficient. The acoustic impedance of a medium is defined by:

$$
Z=\rho c=\sqrt{\frac{\rho}{\kappa}},
$$

where $c$ is the speed of sound in the medium, $\kappa$ is the compressibility, and $\rho$ is the density. 


\section{Scattering}

Scattering is far more complicated [1]: ${ }^{a}$ An inhomogeneity in the path of a sound wave causes it to spread out in a variety of directions [2]. The quotient representing the power scattered per unit solid angle and per unit incident intensity, is referred to as the differential scattering cross section [2]. When the direction toward the receiver extends back to the source, such as in pulse-echo systems, we speak of backscattering. Under this condition, the backscattering cross section equals $4 \pi$ times the differential scattering cross section [2]. When multiplying the differential scattering cross section and the backscattering cross section with the density of the scatterers per unit volume, one obtains the scattering coefficient and the backscattering coefficient, respectively. The backscattering coefficient of a medium containing a small concentration of fluid spheres with radii $r$ is given by [3]:

$$
\eta(\omega)=3 k^{4} \gamma_{0}^{2} r^{3}\left(\frac{J_{1}(2 k r)}{2 k r}\right)^{2}
$$

where $k$ is the acoustic wave number, $J_{1}$ is the first order Bessel function of the first kind, and $\gamma_{0}$ the variance of the density/compressibility fluctuation:

$$
\gamma_{0}^{2}=N_{0} \frac{4 \pi r^{3}}{3}\left(\frac{\kappa_{1}-\kappa_{0}}{\kappa_{0}}-\frac{\rho_{1}-\rho_{0}}{\rho_{0}}\right)^{2}
$$

where $N_{0}$ is the number of scatterers per unit volume, $\kappa_{1}$ is the compressibility of the scatterer, $\kappa_{0}$ is the compressibility of the surrounding medium, $\rho_{1}$ is the density of the scatterer, and $\rho_{0}$ is the density of the surrounding medium.

\footnotetext{
${ }^{a}$ The References in this paper are far but complete. They should, however, provide the reader with sufficient background reading material for further study.
} 
From equations (1) and (4) it follows that blood cells are poor scatterers in the clinical diagnostic frequency range. Since imaging blood flow and measuring organ perfusion are desirable for diagnostic purposes, a marker has to be added to the blood that helps to differentiate between blood and other tissue types, by providing additional and desirably characteristic backscatter [4]. Gas bubbles are suitable markers, not only because they have a high compressibility (gaseous microbubbles are 10,000 times more compressible than red blood cells [5]) and low density compared to the surrounding medium, but also because they resonate. For illustration, De Jong computed the scattering cross section in water of a rigid iron sphere and a gas sphere, both with a $1 \mu \mathrm{m}$ radius [6]. At a driving frequency of $3 \mathrm{MHz}$, the scattering cross section of the gas sphere is $160 \mathrm{~dB}$ higher than that of the rigid sphere.

\section{Ultrasound contrast agents}

Microbubbles that are used for ultrasonic imaging purposes are referred to as ultrasound contrast agents. Their development has gone through several generations [7]. Table 1 gives an overview of the ultrasound contrast agents used most in imaging research. Free microbubbles represent generation 0 . These bubbles rapidly dissolve owing to diffusion.

The change of gas bubble radius as a function of time, due to diffusion, is given by [8]:

$$
\frac{\mathrm{d} r}{\mathrm{~d} t}=D L\left(\frac{\frac{C_{\mathrm{i}}}{C_{0}}-1-\frac{2 \sigma}{r p_{0}}-\frac{p_{\mathrm{s}}^{+}}{p_{0}}}{1+\frac{4 \sigma}{3 r p_{0}}}\right)\left(\frac{1}{r}+\frac{1}{\sqrt{\pi D t}}\right),
$$


where $\frac{C_{\mathrm{i}}}{C_{0}}$ is the ratio of the dissolved gas concentration to the saturation concentration (saturation ratio), $D$ is the diffusion constant, $L$ is the Ostwald coefficient, $p_{0}$ is the ambient pressure, $p_{\mathrm{s}}^{+}$is the applied static overpressure, $r$ is the instantaneous bubble radius, $t$ is the time starting $(t=0)$ when the bubble surface is exposed to the liquid surface, and $\sigma$ is the surface tension. Equation (5) shows that the disappearance of gas bubbles in a liquid medium is highly influenced by gas diffusion parameters and applied overpressure, and that the disappearance time of gas bubbles is shorter when the liquid medium is under pressure. The difference, however, is of a much smaller order than the half-size time of the bubbles (the time it takes for a bubble to dissolve until it has reached half its initial size [9]), as Figure 1 demonstrates.

To prevent them from rapid dissolution, generation 0 microbubbles could have diameters of $80 \mu \mathrm{m}$, which withheld them from passing the lung capillaries. First generation ultrasound contrast agents consist of air bubbles encapsulated by a stabilizing shell. With mean diameters below $6 \mu \mathrm{m}$, these bubbles are small enough to pass through capillaries.

For encapsulated microbubbles, shell stiffness parameters $\chi, S_{\mathrm{sh}}$ have been introduced $[6,10]$ :

$$
\chi=\frac{S_{\mathrm{sh}}}{8 \pi}=\frac{E \epsilon}{1-\nu}
$$

where $E$ is Young's modulus, $\epsilon$ is the shell thickness, and $\nu$ is the Poisson ratio. For albumin and lipid nanoshells, we presume $0.499<\nu<0.500$ [11]. The shell properties of an ultrasound contrast agent have to be derived from experimental data. Second generation agents consist of encapsulated gas microbubbles with 
an elastic shell. The linear angular resonance of microbubbles with a viscous shell can be approximated by a Newtonian model [12, 13]:

$$
\omega_{0}^{2} \approx \frac{3 \Gamma}{r_{0}^{2} \rho}\left(p_{0}+\frac{2 \sigma}{r_{0}}+\frac{2 \chi}{r_{0}}\right)-\frac{2 \sigma+6 \chi}{r_{0}^{3} \rho}
$$

or by a viscoelastic model $[14,15]$ :

$$
\omega_{0}^{2} \approx \frac{1}{r_{0}^{2} \rho}\left(3 \Gamma p_{0}-\frac{4 \sigma}{r_{0}}+\frac{4 E}{r_{0}}\right)
$$

Here, $r_{0}$ is the equilibrium bubble radius, $\Gamma$ is the polytropic exponent of the gas, $\rho$ is the density of the host medium, and $\omega_{0}$ is the angular resonance frequency. Clearly, if the size distribution of the microbubbles is wide, the agent will respond to a wide ultrasonic frequency band. If the size distribution of the microbubbles is narrow, the agent will be selective to a narrow frequency band.

When using perfluorocarbon gases instead of air, the microbubbles will first swell by a factor, because of the diffusion of dissolved gases into the bubbles, and then dissolve. The low diffusion rate of high molecular weight perfluorocarbons prolongs microbubble presence from seconds to minutes [4]. Often, the surface of the bubble shell has a negative charge, to prolong its presence in target tissue $[16]$.

Agents can be designed to specifically target a receptor system [17], thus facilitating ultrasonic molecular imaging [18]. Third generation ultrasound contrast agents consist of bubbles with such special targeting shell properties. Owing to primary radiation forces, microbubbles can be forced to translate away from the transducer, to vessel walls $[18,19]$, increasing the success rate of targeting. 
Submicron contrast agents have also been of interest, because they can travel through the lymphatic system and are small enough to be extravasated from tumor neovasculature $[5,20]$.

Ultrasound contrast agents have been involved in therapeutic applications, as well. Because any drug might in some form be attached to a bubble, the number of potential therapeutic applications of bubbles is virtually unlimited. To limit our scope to diagnostic applications, here we describe the physical mechanisms involved in bubble-imaging techniques. 
Table 1: Overview of the ultrasound contrast agents used most in diagnostic imaging research $[21,22,23,24,25,26,27,28,29,7,30]$. First generation: encapsulated air bubbles, second generation: encapsulated low solubility gas bubbles, third generation: particulated gas bubbles with controlled acoustic properties [31]. This classification is approximate, because not all agents fit neatly into the generation categories.

\begin{tabular}{llll}
\hline Agent & Manufacturor & Shell & Gas/vapor \\
\hline \hline
\end{tabular}

First generation

\begin{tabular}{llllr} 
Albunex & MolecularBiosystems & Albumin & Air & 4.3 \\
Levovist & Schering & Lipid/galactose & Air & $2-3$ \\
Sonovist & Schering & Cyanoacrylate & Air & $1-2$ \\
\hline
\end{tabular}

Second generation

\begin{tabular}{lllll} 
BR14 & Bracco & Lipid & $\mathrm{C}_{4} \mathrm{~F}_{10}$ & $2.5-3.0$ \\
Definity & Bristol-Myers Squibb & Lipid/surfactant & $\mathrm{C}_{3} \mathrm{~F}_{8}$ & $1.1-3.3$ \\
EchoGen & Sonus & Surfactant & $\mathrm{C}_{5} \mathrm{~F}_{12}$ & $2-5$ \\
Imagent & Alliance Pharmaceutic & Lipid/surfactant & $\mathrm{N}_{2} / \mathrm{C}_{6} \mathrm{~F}_{14}$ & 6.0 \\
Optison & GE Healthcare & Albumin & $\mathrm{C}_{3} \mathrm{~F}_{8}$ & $2.0-4.5$ \\
Quantison & Upperton & Albumin & Air & 3.2 \\
SonoVue & Bracco & Lipid & $\mathrm{SF}_{6}$ & 2.5 \\
\hline
\end{tabular}

Third generation

$\begin{array}{lllll}\text { AI-700 } & \text { Acusphere } & \text { PLGA }^{a} & \mathrm{C}_{4} \mathrm{~F}_{10} & 2 \\ \text { CARDIOsphere } & \text { POINT Biomedical } & \text { Polyactide } & \mathrm{N}_{2} & 4.0 \\ \text { Sonazoid } & \text { GE Healthcare } & \text { Lipid/surfactant } & \mathrm{C}_{4} \mathrm{~F}_{10} & 2.4-3.6\end{array}$

${ }^{a}$ Polyactide co-glycolide 
Originally, ultrasound contrast studies were performed for left ventricular function and myocardial perfusion [31]. Nowadays, ultrasound contrast agents have, among others, been implicated in the following diagnostic techniques: imaging the heart [27], vasculature including vasa vasorum, liver, spleen [7], kidneys [32], brain [33, 34], measuring tissue perfusion [4], ejection fractions [35], detecting focal lesions in the liver [36], angiogenesis assessment [37], characterizing tumors, and detecting sites of inflammation [26].

The ultrasound contrast agents currently approved for certain clinical applications are Levovist (Canada, China, Europe, Japan), Definity (Canada, United Kingdom, United States), Optison (Canada, Europe, United States) and SonoVue (China, Europe) [38, 27].

\section{Contrast bubble models}

To enhance detection techniques, predicting the dynamic behavior of ultrasound-insonified encapsulated microbubbles has been of much interest. Their behavior has been studied with acoustic methods such as the measurement of backscattering under different conditions [39], (high-speed) photography $[40,41,42,9]$, and other optical methods, such as the dynamic measurement of light scattering [13].

The oscillating behavior of ultrasound contrast agent microbubbles in Newtonian fluids has been frequently described by a Rayleigh-Plesset-Noltingk-Neppiras-Poritsky (RPNNP) equation modified for the stiffness of a shell $[6,43,44]$ and by a Herring equation modified for a viscous shell [41, 13, 18]. Other models include 
an RPNNP equation modified for the mechanical properties of the shell [45], a zero-thickness interface model [14], and a model accounting for variations in surface tension [46]. Generally, the presence of blood has a relatively small effect on bubble dynamics [47]. The RPNNP equation has been modified to include the shell properties stiffness and friction [48]:

$$
\begin{aligned}
& \rho r \ddot{r}+\frac{3}{2} \rho \dot{r}^{2}=\left(p_{0}+\frac{2 \sigma}{r_{0}}\right)\left(\frac{r_{0}}{r}\right)^{3 \Gamma}-\frac{4 \mu \dot{r}}{r}-\frac{2 \sigma}{r} \\
& -2 \chi\left(\frac{1}{r_{0}}-\frac{1}{r}\right)-\omega^{2} \rho r^{2} \frac{\dot{r}}{c}-\frac{S_{\mathrm{f}} \dot{r}}{4 \pi r^{2}}-\delta_{\mathrm{t}} \omega \rho r \dot{r}-\left(p_{0}+p_{\mathrm{a}}(t)\right),
\end{aligned}
$$

where $c$ is the speed of sound in the medium, $p_{\mathrm{a}}(t)$ is the driving acoustic pressure in time, $S_{\mathrm{f}}$ is the shell friction, $\mu$ is the shear viscosity of the medium, $\omega$ is the angular driving frequency, and $\delta_{\mathrm{t}}$ is the damping coefficient due to heat conduction [49]:

$$
\delta_{\mathrm{t}}=\frac{\frac{\sinh z+\sin z}{\cosh z-\cos z}-\frac{2}{z}}{\frac{z}{3(\Gamma-1)}+\frac{\sinh z-\sin z}{\cosh z-\cos z}},
$$

where $z=r_{0} / l_{\mathrm{D}}$, in which $l_{\mathrm{D}}$ is the thermal boundary layer thickness:

$$
l_{\mathrm{D}}=\sqrt{\frac{K_{\mathrm{g}}}{2 \omega \rho_{\mathrm{g}} C_{\mathrm{p}}}} .
$$

Here, $C_{\mathrm{p}}$ is specific heat of the gas, $K_{\mathrm{g}}$ is the thermal conductivity of the gas, and $\rho_{\mathrm{g}}$ is the density of the gas. Note that the vapor pressure of the liquid has been neglected, which is valid in our situation [50]. The modified RPNNP equation is used for simulating bubble response to insonification at moderate acoustic pressures, i.e. $\mathrm{MI}^{b} \lesssim 0.6[9]$. An example of simulated oscillating behavior of a

\footnotetext{
${ }^{b}$ On clinical ultrasound devices, the intensity of the ultrasonic field is generally adjusted with a switch for the mechanical index instead of the acoustic amplitude. The mechanical index is defined as $\mathrm{MI}=p^{-} / \sqrt{f}$, where $p^{-}$is the peak rarefactional acoustic pressure normalized by $1 \mathrm{MPa}$ and $f$ is the center frequency of the ultrasound normalized by $1 \mathrm{MHz}$.
} 
microbubble is shown in Figure 2. The microbubble with half resonance size oscillates exactly $\phi=\pi \operatorname{rad}$ out of phase with the driving pressure $p_{\mathrm{a}}=p^{+} \sin \omega t$ in which $p^{+}$is the peak-positive acoustic pressure. At resonance, $\phi=\frac{3}{2} \pi \mathrm{rad}$, and at double resonance $\phi=2 \pi \mathrm{rad}$. As the damping becomes less, the transition in offset becomes more abrupt [49]. For microbubbles with thin elastic shells, the modified RPNNP equation gives a slightly conservative estimate of the radial excursion, as opposed to the modified Herring equation [9]. The modified Herring equation is given by $[41,13]$ :

$$
\begin{aligned}
& \rho r \ddot{r}+\frac{3}{2} \rho \dot{r}^{2}=\left(p_{0}+\frac{2 \sigma}{r_{0}}+\frac{2 \chi}{r_{0}}\right)\left(\frac{r_{0}}{r}\right)^{3 \Gamma}\left(1-3 \frac{\dot{r}}{c}\right)-\frac{4 \mu \dot{r}}{r}-\frac{2 \sigma}{r}\left(1-\frac{\dot{r}}{c}\right) \\
& -\frac{2 \chi}{r}\left(\frac{r_{0}}{r}\right)^{2}\left(1-3 \frac{\dot{r}}{c}\right)-12 \epsilon \mu_{\mathrm{s}} \frac{\dot{r}}{r(r-\epsilon)}-\left(p_{0}+p_{\mathrm{a}}(t)\right),
\end{aligned}
$$

where $\mu_{\mathrm{s}}$ is the shell shear viscosity.

Equations (9) and (12) can only be solved numerically. Figure 3 shows solutions of the modified Herring equation and Fourier-spectra thereof, at different acoustic amplitudes, for a bubble with a negligible shell. At $10 \mathrm{kPa}$ the bubble oscillates linearly, but at higher acoustic amplitudes the bubble has a longer expansion than a contraction phase, and a higher outward than inward excursion. At $391 \mathrm{kPa}$, a slow expansion is followed by a rapid collapse, which is followed by a number of rebounds. Furthermore, the maximal bubble excursion is more than twelve times the equilibrium radius. The nonlinear behavior described here generates harmonics. Examples of the acoustic emission from ultrasound contrast agent bubbles can be appreciated in [20]. Figure 4 demonstrates the spectra of the ultrasound contrast agent Levovist, insonified at different driving 
amplitudes [34]. Similar results were presented in [51]. The quasi-continuous part in the spectrum of the acoustic response to the highest driving amplitude has been attributed to microbubble disruption, which is discussed in a following section.

Figure 5 shows $r-t$ curves computed with both models, for a free gas microbubble, and for an encapsulated microbubble, insonified at a relatively high acoustic pressure of $210 \mathrm{kPa}$. Both for the free gas bubble and for the encapsulated bubble, the curves are very similar.

\section{Harmonic imaging}

At lower driving pressures, microbubbles produce linear backscatter enhancement, only resulting in an augmentation of the echo from blood [31]. By making use of nonlinear behavior of microbubbles resulting in harmonic backscatter, blood can be discriminated from the surrounding tissue. The increase of nonlinear behavior with acoustic driving pressure has been demonstrated in Figures 3-5. To suppress signal from tissue, a band pass filter around the second harmonic frequency component of the echo can be used to produce the ultrasound image [52]. This imaging technique is ineffective, due to the wide overlap of the baseband and the second harmonic in broadband systems. Furthermore, this technique is not effective at high driving pressures, since tissue harmonics may interfere with the signals from the perfused areas. Therefore, nondestructive imaging is preferably done at moderate acoustic pressures.

Given the lack of subharmonic generation in tissue, subharmonic imaging is 
an alternative [53]. However, subharmonic generation occurs only, when the acoustic amplitude exceeds a certain threshold level. Goertz et al. recently demonstrated that microvessels can be successfully detected in vivo using subharmonic imaging with a transmit frequency of $20 \mathrm{MHz}$ [54].

Multipulse techniques have been developed to allow for the separation of contrast agent signal from tissue signal [52]. In these techniques, multiple pulses with modifications in amplitude (power modulation) [55, 56], phase (pulse inversion) $[57,58,56]$, or pulse length (pulse subtraction) [59] are transmitted rapidly after one another, after which the linear contribution in the signal echoes is canceled out by summation or subtraction, leaving a residual signal containing harmonics ( $c f$. Figure 6). Overviews of multipulse imaging methods have been given in $[58,60,59]$. With the introduction of affordable broadband transducers, far more complicated pulse schemes have become possible, such as multifrequency excitation [59, 61]. Since the bubble oscillation amplitude depends on the ambient pressure, a relatively low frequency can be used as if to modulate the ambient pressure, whereas a frequency close to bubble resonance is used to excite the bubble. Two-frequency excitation may be sufficient to induce nonlinear behavior of the microbubbles at moderate incident pressures [59]. Coded excitation, another technique, operates by transmitting a relatively long pulse (high energy) with a low amplitude (low destruction rate), that is decoded (compressed) to obtain sufficient axial resolution. The use of chirps, long bursts with increasing or decreasing instantaneous frequency, has been proposed for coded excitation [59]. 


\section{Microbubble disruption}

During the collapse phase, when the kinetic energy of the bubble surpasses its surface energy, a bubble may fragment into a number of smaller bubbles [42, 62, 63]. Fragmentation has been exclusively observed with contrast agents with thin elastic shells. For these bubbles, fragmentation is the dominant disruption mechanism [62].

Thick-shelled bubbles have demonstrated gas release during a high-amplitude ultrasonic cycle $[64,9,65]$. The increased pressure difference between inside and outside of the bubble during the ultrasonic wave causes the shell to be stretched across a critical deformation, resulting into its mechanical cracking. The released bubble has an oscillation amplitude much higher than an encapsulated bubble of the same size. Therefore, the acoustic signal from a contrast agent after gas release differs from that of the same contrast agent before gas release, until the released gas has dissolved.

Although the coalescence (merge) of ultrasound contrast agent microbubbles has been observed in vitro, this phenomenon is negligible in vivo, because long low-amplitude bursts are needed for forced approach of microbubbles [9].

Ultrasound-induced disruption of the microbubbles will result in strong, transient harmonic echoes [66]. These strong echoes reveal instantly which areas on the resulting image represent perfused regions. After a disruptive ultrasonic burst, the disappearance of microbubble fragments or released gas may be traced with low-amplitude ultrasound, as well as the wash-in rate of fresh contrast agent [67]. With the pressure-dependant changes in the echo signal, hydrostatic 
overpressures might be determined. Making use of the subharmonic response leads to more precise sizing $[9,68]$ and to more precise pressure measurements $[44,69]$.

\section{Expert opinion}

According to the World Health Organization, in $20-30 \%$ of cases worldwide, clinical considerations alone are not sufficient to come to a correct diagnosis [70]. Of these cases, 80-90\% can be diagnosed using common X-ray or ultrasound examinations [70]. The most performed diagnostic imaging technology is X-ray, followed at a distance by ultrasound. For example, in Ontario, Canada, 63\% of the diagnostic imaging examinations in hospitals are done with X-ray, $16 \%$ with ultrasound, $9 \%$ with computed tomography, $7 \%$ with nuclear medicine, $4 \%$ with magnetic resonance imaging, and $1 \%$ with catheterization [71]. When taking into account the absolute hospital operating expenses [71], X-ray and ultrasound have approximately the same price per examination. Other imaging techniques are roughly three times as expensive, except for catheterization, which is twenty times as expensive. However, X-ray is a less desirable imaging technique than ultrasound, due to the negative radiation effects. Therefore, novel ultrasound-based imaging techniques are being developed that may compete with other imaging techniques.

The main disadvantage of ultrasonography has been that only physical properties of the tissue are imaged. A new generation of ultrasound contrast agents may help to overcome this limitation, with ligands having specific 
targeting properties attached to the shell of the microbubbles. Such agents would certainly be more expensive than the current second generation agents. But, by providing accurate and reliable early diagnosis, contrast sonography could reduce downstream resource use and with that overall health care costs, justifying incremental examination costs [4].

\section{Five-year view}

Ultrasonic contrast-enhanced imaging applications depend on the detectability of microbubbles. The detectability in turn depends on the ultrasonic pulsing scheme. Initiatives have been undertaken to find the optimal pulse sequence for a maximal contrast-to-tissue-ratio in combination with an imaging resolution as high as possible. Enhancement in image quality owing to coded excitation has been anticipated in the near future.

Note that ultrasound contrast agent bubble responses will become better predictable, if the bubble populations have a narrower size distribution and uniform shell thicknesses.

Ultrasound images acquired using lipid-shelled microbubbles targeted to leukocytes were presented in [72]. The high resolution of these in vivo images in comparison to the ex vivo gamma camera images of the same tissue samples is striking. The potential of targeted ultrasonic imaging is clearly demonstrated.

Ultrasound-induced release of therapeutic substances from microbubbles has been proposed in numerous papers $[73,74,75]$. Combining diagnostic, targeted microbubbles with therapeutic substances may lead to a simple method to 
instantly cure upon diagnosis.

Contrast agents for magnetic resonance (MR) imaging have been designed to accumulate in specific cells $[1,76]$. As opposed to ultrasound contrast agents, whose microbubbles are limited to flowing through vessels, MR contrast agents consist of nanoparticles small enough to access cells. Undesirable accumulation of MR contrast agent takes place in organs other than the imaged, too. Nanometer-sized magnetic resonance markers may be coupled to microbubbles. In the organ to be imaged, these markers are to be released with a high-MI ultrasonic burst. This will result in an increase of the uptake MR contrast agent by the targeted organ. Radionuclides used for positron emission tomography might also be encapsulated by microbubbles, to reduce unspecific binding.

Increasing concern has been shown for ultrasound safety issues when using ultrasound contrast agents [77]. Although no side effects have been officially reported [4], current acoustic amplitudes allowed are based on tissue without cavitation nuclei, such as microbubbles, present. Since the use of ultrasound contrast agents has become increasingly popular, and their applications have been multiplied, a new safety standard in ultrasonic imaging will have to be defined in the near future.

In conclusion, the authors think that ultrasound is becoming an even more important technique in clinical diagnostics.

\section{Key issues}

- Predicting the transient behavior of microbubbles. 
- Improving detection presence of bubbles by:

improving pulsing schemes,

improving processing methods,

improving bubble properties.

- Combining ultrasonic imaging with other techniques, such as magnetic resonance imaging and positron emission tomography.

- Defining a new safety standard in ultrasonic imaging.

\section{References $^{c}$}

[1] Webb A. Introduction to Biomedical Imaging. John Wiley \& Sons, Hoboken (2003).

[2] Pierce AD. Acoustics: An Introduction to Its Physical Principles and Applications. Acoustical Society of America, New York (1989).

[3] Schmitz G. Ultrasound in medical diagnosis. In: Pike R, Sabatier P (Eds.), Scattering: scattering and inverse scattering in pure and applied science, Academic Press, London (2002).

[4] Schutt EG, Klein DH, Mattrey RM, Riess JG. Injectable microbubbles as contrast agents for diagnostic ultrasound imaging: the key role of perfluorochemicals. Angew. Chem. Int. Ed. 42, 3218-3235 (2003).

\footnotetext{
${ }^{c} \bullet$ : of interest, $\bullet \bullet$ : of considerable interest.
} 
[5] Patel DN, Bloch SH, Dayton PA, Ferrara KW. Acoustic signatures of submicron contrast agents. IEEE Trans. Ultrason., Ferroelect., Freq. Contr. 51(3), 293-301 (2004).

[6] de Jong N. Acoustic properties of ultrasound contrast agents. Ph.D. thesis, Erasmus Universiteit Rotterdam (1993).

[7] Krestan C. Ultraschallkontrastmittel: Substanzklassen, Pharmakokinetik, klinische Anwendungen, Sicherheitsaspekte. Radiologe 45, 513-519 (2005).

[8] Bouakaz A, Frinking PJA, de Jong N, Bom N. Noninvasive measurement of the hydrostatic pressure in a fluid-filled cavity based on the disappearance time of micrometer-sized free gas bubbles. Ultrasound Med. Biol. 25(9), 1407-1415 (1999).

- Presents simulations and acoustic measurements of the dissolution of gas bubbles.

[9] Postema MAB. Medical Bubbles. Ph.D. thesis, Universiteit Twente (2004).

[10] Gorce JM, Arditi M, Schneider M. Influence of bubble size distribution on the echogenicity of ultrasound contrast agents: A study of SonoVue ${ }^{\mathrm{TM}}$. Invest. Radiol. 35(11), 661-671 (2000).

[11] Postema M, de Jong N, Schmitz G. Nonlinear behavior of ultrasound-insonified encapsulated microbubbles. Proc. 17th Int. Symp. Nonlinear Acoustics in press (2005). 
[12] MacDonald CA, Sboros V, Gomatam J, Pye SD, Moran CM, McDicken WN. A numerical investigation of the resonance of gas-filled microbubbles: resonance dependence on acoustic pressure amplitude. Ultrasonics 43, $113-122(2004)$.

[13] Guan J, Matula TJ. Using light scattering to measure the response of individual ultrasound contrast microbubbles subjected to pulsed ultrasound in vitro. J. Acoust. Soc. Am. 116(5), 2832-2842 (2004).

[14] Sarkar K, Shi WT, Chatterjee D, Forsberg F. Characterization of ultrasound contrast microbubbles using in vitro experiments and viscous and viscoelastic interface models for encapsulation. J. Acoust. Soc. Am. 118(1), 539-550 (2005).

[15] Tickner EG. The resonant frequency of a bubble with a thin, surrounding membrane. Technical report, POINT Biomedical Corporation (2005).

[16] Fisher NG, Christiansen JP, Klibanov A, Taylor RP, Kaul S, Lindner JR. Influence of microbubble surface charge on capillary transit and myocardial contrast enhancement. J. Am. Coll. Cardiol. 40(4), 811-819 (2002).

[17] Lathia JD, Leodore L, Wheatley MA. Polymeric contrast agent with targeting potential. Ultrasonics 42, 763-768 (2004).

[18] Zhao S, Borden M, Bloch SH, Kruse D, Ferrara KW, Dayton PA. Radiation-force assisted targeting facilitates ultrasonic molecular imaging. Mol. Imaging 3(3), 135-148 (2004). 
[19] Tortoli P, Boni E, Corsi M, Arditi M, Frinking P. Different effects of microbubble destruction and translation in Doppler measurements. IEEE Trans. Ultrason., Ferroelect., Freq. Contr. 52(7), 1183-1188 (2005).

[20] Zheng H, Mukdadi O, Shandas R. Theoretical predicitions of harmonic generation from submicron ultrasound contrast agents for nonlinear biomedical ultrasound imaging. Phys. Med. Biol. 51, 557-573 (2006).

[21] Frinking PJA, de Jong N. Acoustic modeling of shell-encapsulated gas bubbles. Ultrasound Med. Biol. 24(4), 523-533 (1998).

[22] Forsberg F, Goldberg BB, Liu JB, Merton DA, Rawool NM, Shi WT. Tissue-specific US contrast agent for evaluation of hepatic and splenic parenchyma. Radiology 210, 125-132 (1999).

[23] Sontum PC, Østensen J, Dyrstad K, Hoff L. Acoustic properties of NC100100 (Sonazoid ${ }^{\mathrm{TM}}$ ) and their relationship with the microbubble size distribution: motivation for choice of assay and dosage parameter. Proc. IEEE Ultrason. Symp. 1743-1748 (1999).

[24] Heckemann RA, Harvey CJ, Blomley MJK, Eckersley RJ, Butler-Barnes J, Jayaram V, Cosgrove DO. Enhancement characteristics of the microbubble agent Levovist: reproducibility and interaction with aspirin. Eur. J. Radiol. 41, 179-183 (2002).

[25] Camarano G, Jones M, Freidlin RZ, Panza JA. Quantitative assessment of left ventricular perfusion defects using real-time three-dimensional 
myocardial contrast echocardiography. J. Am. Soc. Echocardiogr. 15(3), $206-213$ (2002).

[26] Lindner JR. Microbubbles in medical imaging: current applications and future directions. Nature Rev. Drug Discov. 3, 527-532 (2004).

[27] Miller AP, Nanda NC. Contrast echocardiography: new agents. Ultrasound Med. Biol. 30(4), 425-434 (2004).

[28] Yang L. Real-time myocardial contrast echocardiography and its applications in evaluation for coronary artery disease. Chin. Med. J. 117(9), 1388-1394 (2004).

[29] Raisinghani A, Rafter P, Phillips P, Vannan MA, DeMaria AN. Microbubble contrast agents for echocardiography: rationale, composition, ultrasound interactions, and safety. Cardiol. Clin. 22, 171-180 (2004).

[30] Kollmann C, Putzer M. Ultraschallkontrastmittel — physikalische Grundlagen. Radiologe 45(6), 503-512 (2005).

[31] Becher H, Burns PN. Handbook of Contrast Echocardiography: LV Function and Myocardial Perfusion. Springer-Verlag, Berlin (2000).

-• Presents an excellent overview of contrast echocardiography.

[32] Heynemann H, Jenderka KV, Zacharias M, Fornara P. Neue Techniken der Urosonographie. Urologe 43, 1362-1370 (2004). 
[33] Droste DW, Kaps M, Navabi DG, Ringelstein EB. Ultrasound contrast enhancing agents in neurosonology: principles, methods, future possibilities. Acta Neurol. Scand. 102, 1-10 (2000).

[34] Wilkening WG. Konzepte zur Signalverarbeitung für die kontrastmittelspezifische Ultraschallabbildung. Ph.D. thesis, Ruhr-Universität Bochum (2003).

[35] Mischi M, Jansen AHM, Kalker AACM, Korsten HHM. Identification of ultrasound contrast agent dilution systems for ejection fraction measurements. IEEE Trans. Ultrason., Ferroelect., Freq. Contr. 52(3), $410-420(2005)$.

[36] Sidhu PS. Microbubbles are here to burst! Radiol. Now 20, 2-3 (2003).

[37] Heppner P, Lindner JR. Contrast ultrasound assessment of angiogenesis by perfusion and molecular imaging. Expert Rev. Mol. Diagn. 5(3), 447-455 (2005).

[38] Bruce M, Averkiou M. Imaging ultrasound contrast agents. Presented at AAPM 46th Annu. Meet. (2004).

[39] Sboros V, Pye SD, MacDonald CA, Gomatam J, Moran CM, McDicken WN. Absolute measurement of ultrasonic backscatter from single microbubbles. Ultrasound Med. Biol. 31(8), 1063-1072 (2005).

[40] de Jong N, Frinking PJA, Bouakaz A, Goorden M, Schuurmans T, Jingping $\mathrm{X}$, Mastik F. Optical imaging of contrast agent microbubbles in an 
ultrasound field with a 100-MHz camera. Ultrasound Med. Biol. 26(3), 487-492 (2000).

[41] Morgan KE, Allen JS, Dayton PA, Chomas JE, Klibanov AL, Ferrara KW. Experimental and theoretical evaluation of microbubble behavior: Effect of transmitted phase and bubble size. IEEE Trans. Ultrason., Ferroelect., Freq. Contr. 47(6), 1494-1509 (2000).

•• Presents a Herring model modified for encapsulated microbubbles.

[42] Chomas JE, Dayton P, May D, Ferrara K. Threshold of fragmentation for ultrasonic contrast. J. Biomed. Opt. 6(2), 141-150 (2001).

[43] Church CC. The effects of an elastic solid surface layer on the radial pulsations of gas bubbles. J. Acoust. Soc. Am. 97(3), 1510-1521 (1995).

[44] Ganor Y, Adam D, Kimmel E. Time and pressure dependence of acoustic signals radiated from microbubbles. Ultrasound Med. Biol. 31(10), 1367-1374 (2005).

[45] Stride E, Saffari N. On the destruction of microbubble ultrasound contrast agents. Ultrasound Med. Biol. 29(4), 563-573 (2003).

[46] Marmottant P, van der Meer S, Versluis M, de Jong N, Hilgenfeldt S, Lohse D. A model for large amplitude oscillations of coated bubbles accounting for buckling and rupture. Abstr. 10th Eur. Symp. Ultrasound Contrast Imaging $23-33(2005)$. 
[47] Stride E, Saffari N. Theoretical and experimental investigation of the behaviour of ultrasound contrast agent particles in whole blood. Ultrasound Med. Biol. 30(11), 1495-1509 (2004).

[48] de Jong N, Bouakaz A, Frinking P. Basic acoustic properties of microbubbles. Echocardiography 19(3), 229-240 (2002).

[49] Postema M, de Jong N, Schmitz G. The physics of nanoshelled microbubbles. Biomed. Tech. 50(S1), 748-749 (2005).

[50] Young FR. Cavitation. McGraw-Hill, Maidenhead (1989).

[51] Shi WT, Forsberg F, Raichlen JS, Needleman L, Goldberg BB. Pressure dependence of subharmonic signals from contrast microbubbles. Ultrasound Med. Biol. 25(2), 275-283 (1999).

[52] Frinking PJA. Technological review of contrast-enhanced ultrasonography. In: Albrecht T, D’Onofrio M, Frauscher F, Frinking PJA, Lee SH, Nicolau C, Sidhu PS (Eds.), Contrast-Enhanced General Purpose Ultrasound, Springer-Verlag, Milan, 1-8 (2005).

[53] Forsberg F, Shi WT, Goldberg BB. Subharmonic imaging of contrast agents. Ultrasonics 38, 93-98 (2000).

[54] Goertz DE, Cherin E, Needles A, Karshafian R, Brown AS, Burns PN, Foster FS. High frequency nonlinear B-scan imaging of microbubble contrast agents. IEEE Trans. Ultrason., Ferroelect., Freq. Contr. 52(1), 65-79 (2005). 
[55] Christopher T. Finite amplitude distortion-based inhomogeneous pulse echo ultrasonic imaging. IEEE Trans. Ultrason., Ferroelect., Freq. Contr. 44(1), 125-139 (1997).

[56] Eckersley RJ, Chin CT, Burns PN. Optimising phase and amplitude modulation schemes for imaging microbubble contrast agents at low acoustic power. Ultrasound Med. Biol. 31(2), 213-219 (2005).

[57] Hope Simpson D, Chin CT, Burns PN. Pulse inversion Doppler: a new method for detecting nonlinear echoes from microbubble contrast agents. IEEE Trans. Ultrason., Ferroelect., Freq. Contr. 46(2), 372-382 (1999).

[58] Hope Simpson D. Detecting and Imaging Microbubble Contrast Agents With Ultrasound. Ph.D. thesis, University of Toronto (2000).

[59] Borsboom JMG. Advanced Detection Strategies for Ultrasound Contrast Agents. Ph.D. thesis, Erasmus Universiteit Rotterdam (2005).

•• Presents novel detection methods.

[60] Chin CT. Modelling the Behaviour of Microbubble Contrast Agents for Diagnostic Ultrasound. Ph.D. thesis, University of Toronto (2001).

[61] Zheng H, Mukdadi O, Kim H, Hertzberg JR, Shandas R. Advantages in using multifrequency excitation of contrast microbubbles for enhancing echo particle image velocity techniques: initial numerical studies using rectangular and triangular waves. Ultrasound Med. Biol. 31(1), 99-108 (2005). 
[62] Postema M, Bouakaz A, Chin CT, de Jong N. Optical observations of ultrasound contrast agent destruction. Acta Acust. united Acust. 89, 728 (2003).

[63] Yeh CK. Ultrasonic Quantitative Blood Flow Estimation. Ph.D. thesis, National Taiwan University (2004).

[64] Bloch SH, Wan M, Dayton PA, Ferrara KW. Optical observation of lipidand polymer-shelled ultrasound microbubble contrast agents. Appl. Phys. Lett. 84(4), 631-633 (2004).

[65] Prentice P, Cuschieri A, Dholakia K, Prausnitz M, Campbell P. Membrane disruption by optically controlled microbubble cavitation. Nature Phys. 1, $107-110(2005)$.

- Presents a method to control and study bubbles near cells.

[66] Ammi AY, Cleveland RO, Mamou J, Wang GI, Bridal SL, O’Brien Jr WD. Shelled ultrasound contrast agent rupture identification by inertial cavitation and rebound signals. Ultrasonic Imaging 26(4), 257-258 (2004).

[67] Moran CM, Anderson T, Pye SD, Sboros V, McDicken WN. Quantification of microbubble destruction of three fluorocarbon-filled ultrasonic contrast agents. Ultrasound Med. Biol. 26(4), 629-639 (2000).

[68] Biagi E, Breschi L, Masotti L. Transient subharmonic and ultraharmonic acoustic emission during dissolution of free gas microbubbles. IEEE Trans. Ultrason., Ferroelect., Freq. Contr. 52(6), 1048-1054 (2005). 
[69] Adam D, Sapunar M, Burla E. On the relationship between encapsulated ultrasound contrast agent and pressure. Ultrasound Med. Biol. 31(5), 673-686 (2005).

[70] World Health Organization. Essential diagnostic imaging (flyer) (http://www.who.int/entity/eht/en/DiagnosticImaging.pdf).

[71] Canadian Institute for Health Information. Medical imaging in Canada (2004).

[72] Christiansen JP, Leong-Poi H, Klibanov AL, Kaul S, Lindner JR. Noninvasive imaging of myocardial reperfusion injury using leukocyte-targeted contrast echocardiography. Circulation 105, 1764-1767 (2002).

[73] May D, Allen J, Gut J, Ferrara K. Acoustic fragmentation of therapeutic contrast agents designed for localized drug delivery. Proc. IEEE Ultrason. Symp. 1385-1388 (2001).

[74] Shortencarier MJ, Dayton PA, Bloch SH, Schumann PA, Matsunaga TO, Ferrara KW. A method for radiation-force localized drug delivery using gas-filled lipospheres. IEEE Trans. Ultrason., Ferroelect., Freq. Contr. 51(7), 822-831 (2004).

[75] Postema M, Bouakaz A, ten Cate F, Schmitz G, de Jong N, van Wamel A. Nitric oxide delivery by ultrasonic cracking: some limitations. Ultrasonics in press (2006). 
[76] Reimer P, Balzer T. Ferucarbotran (Resovist): a new clinically approved RES-specific contrast agent for contrast-enhanced MRI of the liver: properties, clinical development, and applications. Eur. Radiol. 13, 1266-1276 (2003).

[77] DGBMT, DEGUM, DRG. Ultraschall in der Medizin: Grundlegende Aspekte zur sicheren Anwendung von Ultraschall in der Diagnostik. Deutsche Gesellschaft für Biomedizinische Technik im VDE, Frankfurt am Main (2004).

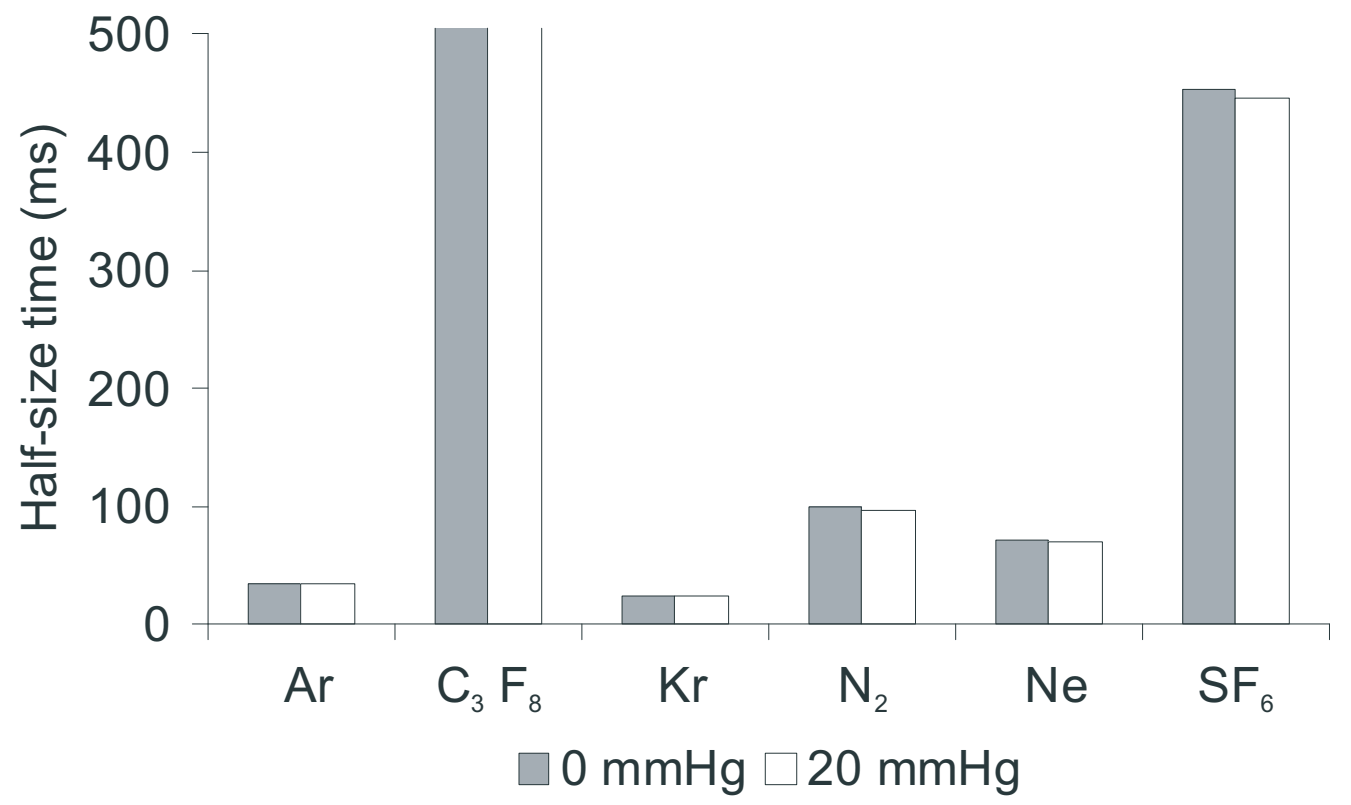

Figure 1: Half-size times of $r_{0}=3 \mu \mathrm{m}$ bubbles containing different gases, at ambient pressure and at an overpressure of $20 \mathrm{mmHg}$. The saturation ratio was taken $\frac{C_{\mathrm{i}}}{C_{0}}=0$ [9]. The half-size time of $\mathrm{C}_{3} \mathrm{~F}_{8}$ exceeds $1000 \mathrm{~ms}$. The difference in dissolving time between 0 and $20 \mathrm{mmHg}$ is on the order of $1 \mathrm{~ms}$. 

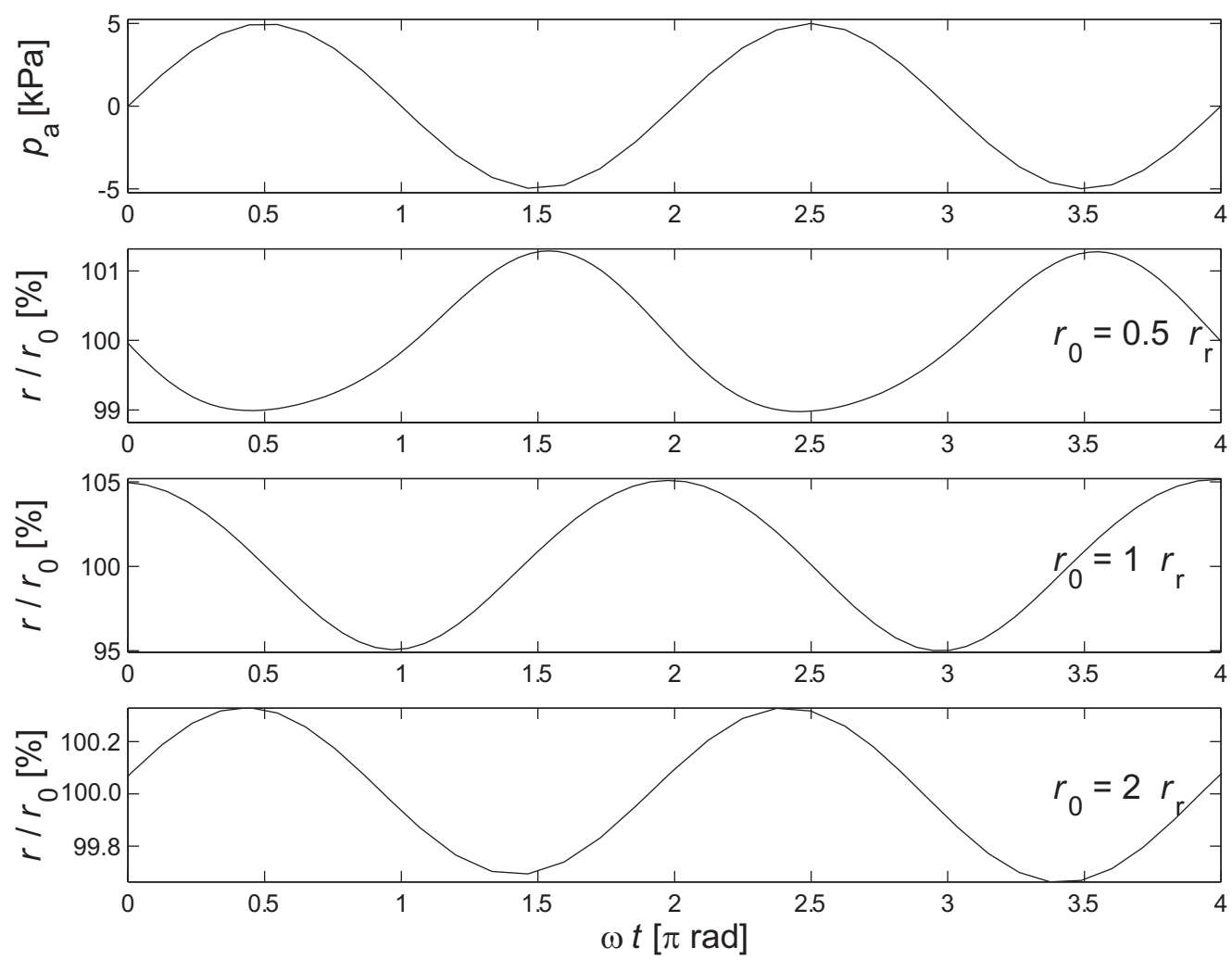

Figure 2: Simulations of the relative bubble surface excursion as a function of the driving phase for underdamped microbubbles with equilibrium radii $r_{0}=\frac{1}{2} r_{\mathrm{r}}, 1 r_{\mathrm{r}}, 2 r_{\mathrm{r}}$, where $r_{\mathrm{r}}$ is the resonant radius, during insonification at $\omega / 2 \pi=0.5 \mathrm{MHz}$ and $p^{+}=5 \mathrm{kPa}$. (C) 2005 Schiele \& Schön GmbH. Reprinted with permission from Postema M, de Jong N, Schmitz G. The physics of nanoshelled microbubbles. Biomed. Tech. 50(S1), 748-749 (2005). 

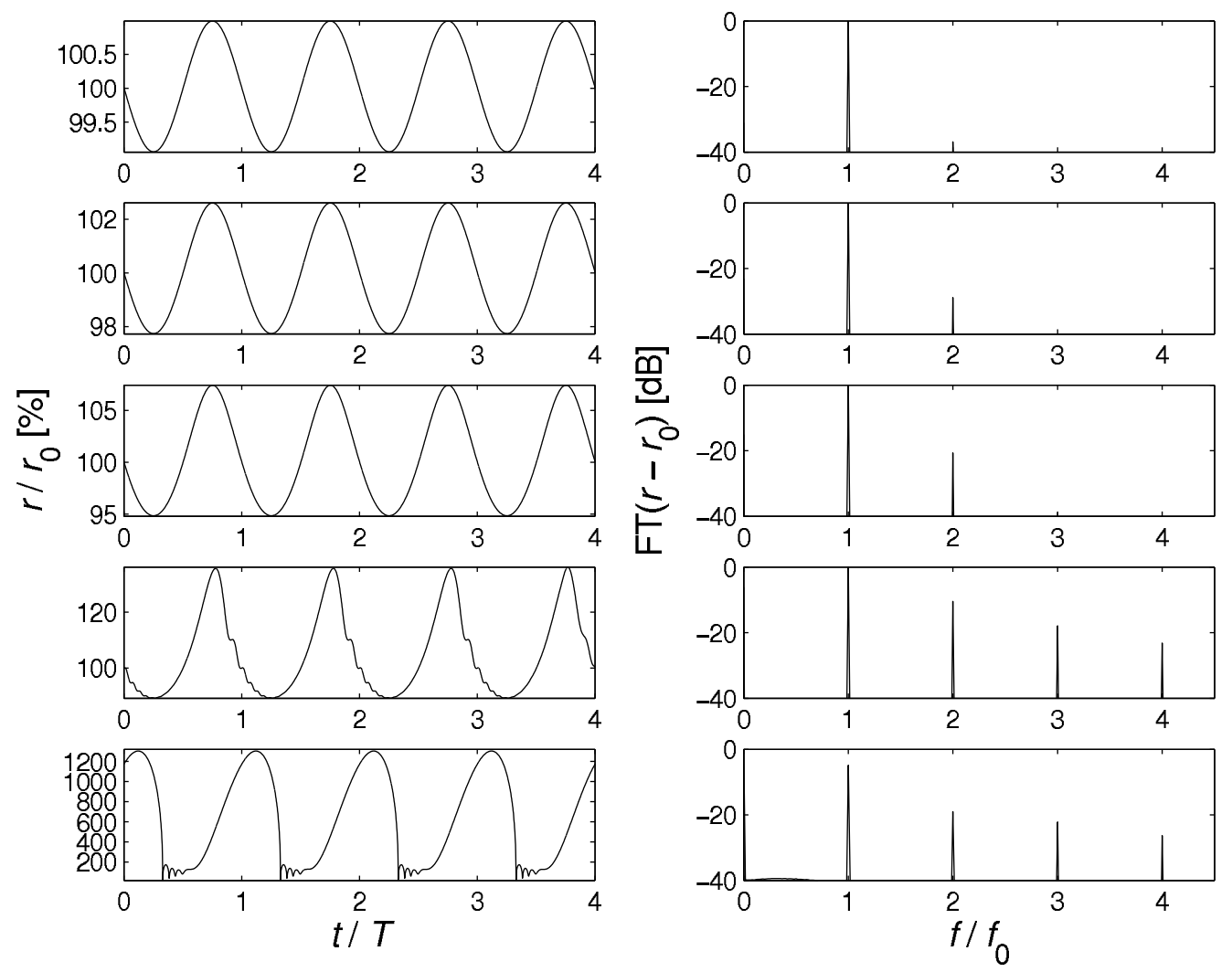

Figure 3: Computed solutions of the modified Herring equation and Fourier-spectra thereof for a bubble with a negligible shell (i.e. $\mu_{\mathrm{s}}=\mu$ and $\chi=0 \mathrm{~kg} \mathrm{~s}^{-2}$ ), at five different acoustic amplitudes, taking $c=1480 \mathrm{~m} \mathrm{~s}^{-1}$, $\omega / 2 \pi=0.5 \mathrm{MHz}, p_{0}=1 \mathrm{~atm}, r_{0}=0.75 \mu \mathrm{m}, \mu=10^{-3} \mathrm{Pas}, \rho=998 \mathrm{~kg} \mathrm{~m}^{-3}$, and $\sigma=0.072 \mathrm{~N} \mathrm{~m}^{-1}$. The acoustic amplitudes were $10.0,25.0,62.5,156$, and $391 \mathrm{kPa}$, respectively. Clearly, the harmonic components increase with the acoustic amplitude. 

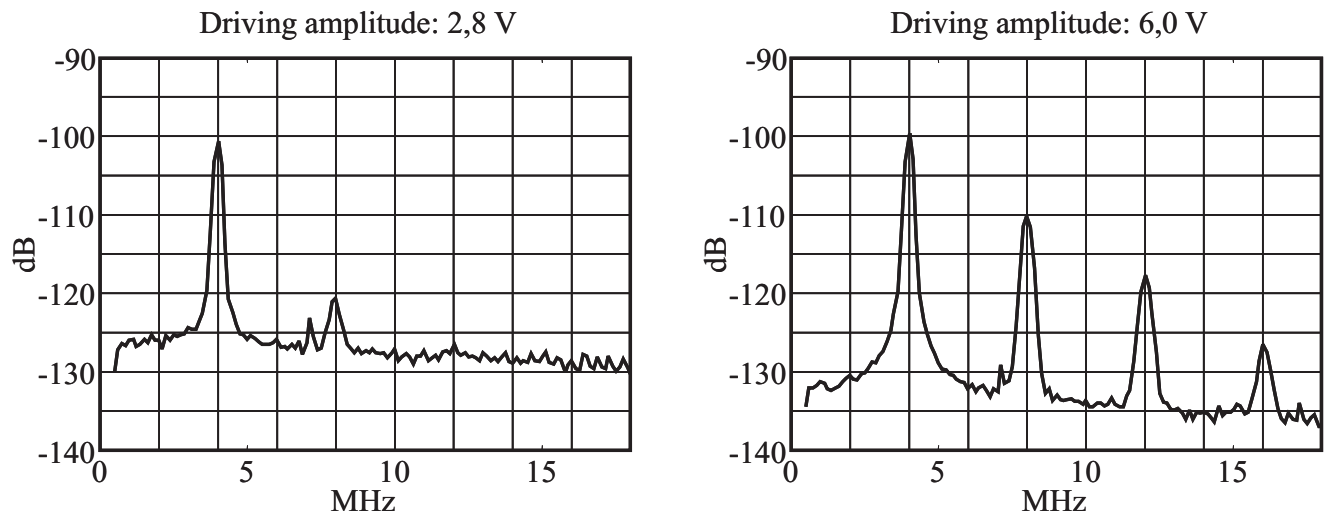

Driving amplitude: $19,1 \mathrm{~V}$
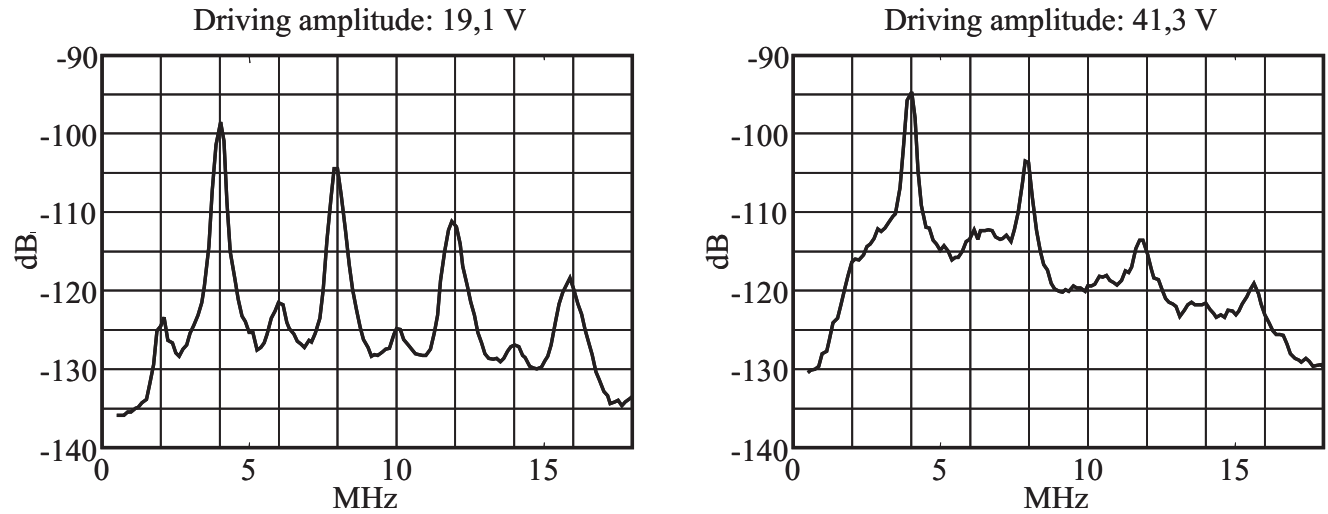

Figure 4: Measurements on a Levovist sample. The driving pulse was a narrow-band Gaussian pulse with a center frequency of $4 \mathrm{MHz}$ and a $-20 \mathrm{~dB}$ bandwidth of $512 \mathrm{~Hz}$. The spectra of the acoustic responses were normalized by the respective driving amplitudes. The echo-amplitude rises out of proportion with the driving amplitude. Moreover, higher harmonics, subharmonics, and ultraharmonics appear. At the highest driving amplitude, the spectrum appears quasi-continuous, with a shift of the higher harmonics to lower frequencies. (c) 2003 Wilko G. Wilkening. Reprinted with permission from Wilkening WG. Konzepte zur Signalverarbeitung für die kontrastmittelspezifische Ultraschallabbildung. Ph.D. thesis, Ruhr-Universität Bochum (2003). 

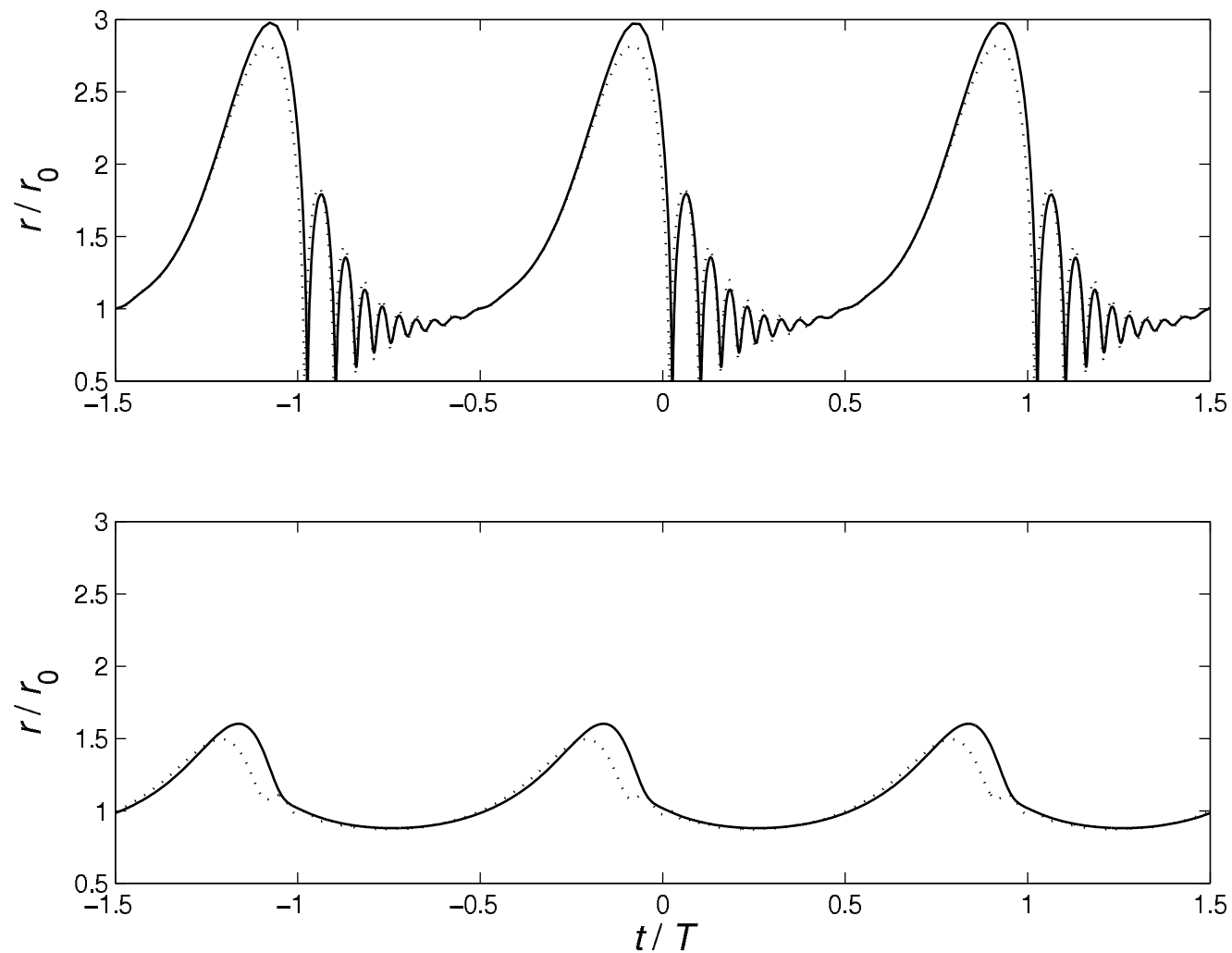

Figure 5: Computed solutions of the modified RPNNP equation (dotted line) and the modified Herring equation (bold line), taking $c=1480 \mathrm{~m} \mathrm{~s}^{-1}, \omega / 2 \pi=$ $0.5 \mathrm{MHz}, p_{0}=1 \mathrm{~atm}, p^{+}=210 \mathrm{kPa}, r_{0}=0.75 \mu \mathrm{m}, \epsilon=20 \mathrm{~nm}, \mu=10^{-3} \mathrm{Pas}$, $\rho=998 \mathrm{~kg} \mathrm{~m}^{-3}$, and $\sigma=0.072 \mathrm{~N} \mathrm{~m}^{-1}$. The upper frame shows $r-t$ curves of a free gas bubble, with $S_{\mathrm{f}}=0 \mathrm{~kg} \mathrm{~s}^{-1}, \delta_{\mathrm{t}}=0, \mu_{\mathrm{s}}=\mu \mathrm{Pas}$, and $\chi=0 \mathrm{~kg} \mathrm{~s}^{-2}$. The lower frame shows $r-t$ curves of an encapsulated bubble with a thin, elastic shell, with $S_{\mathrm{f}}=0 \mathrm{~kg} \mathrm{~s}^{-1}, \delta_{\mathrm{t}}=0, \mu_{\mathrm{s}}=10^{-1} \mathrm{Pas}$, and $\chi=\frac{1.1}{8 \pi} \mathrm{kg} \mathrm{s}^{-2}$. The shell stiffness $\chi$ has been determined in [10]. 

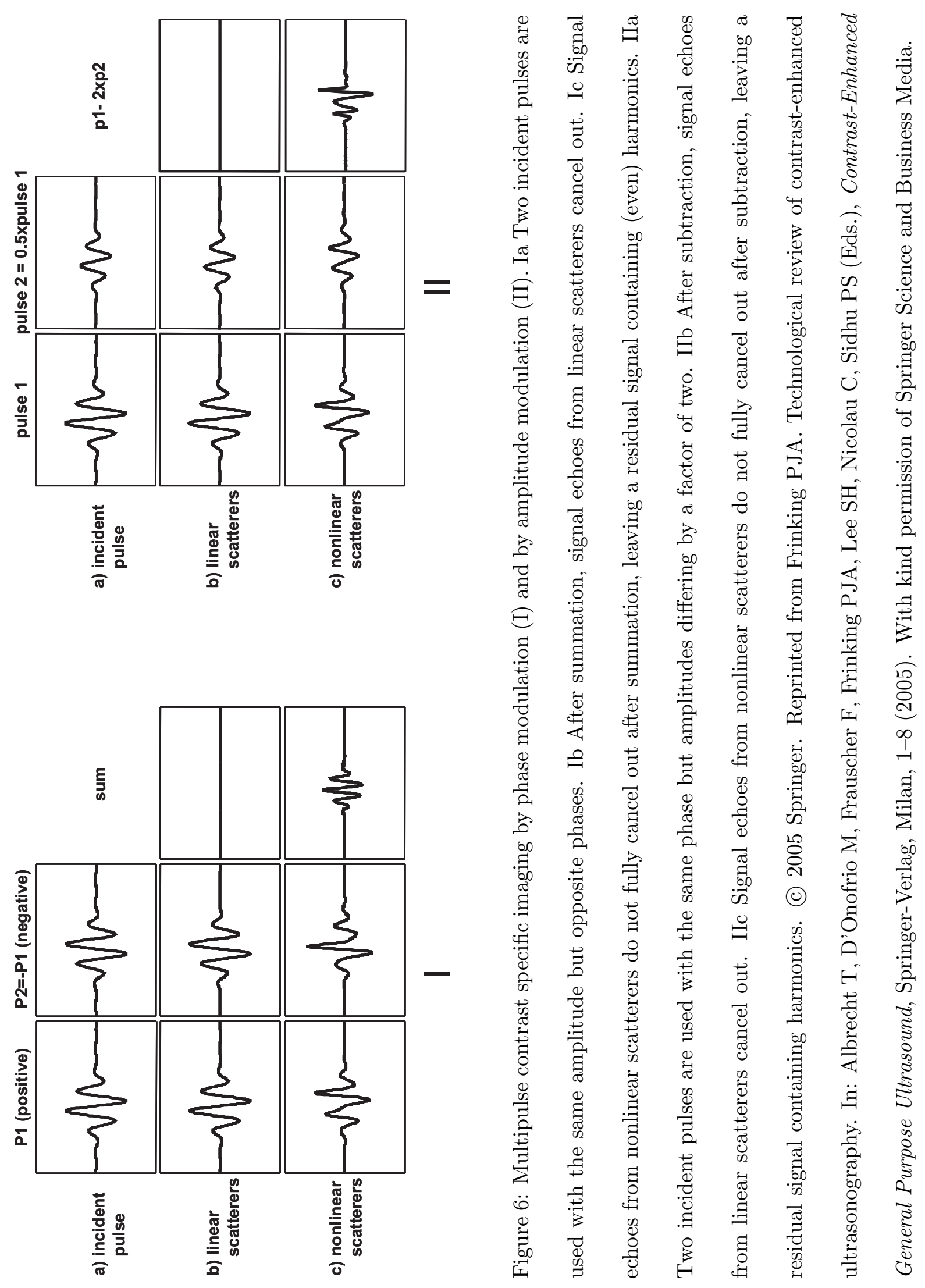